\title{
A new proactive virtual resource center navigation model identifies patient risk factors to reduce barriers to cancer care during the COVID-19 pandemic
}

\author{
Sharon M. Bigelow ${ }^{1}$ (D) Elizabeth Hart ${ }^{1} \cdot \operatorname{Tina}_{\text {Shaban }}{ }^{1} \cdot$ Preeyanka Rao $^{2} \cdot$ Ali Ahmad Khan $^{2} \cdot$ Marianne Baskaron $^{1}$. \\ Pamela Baker ${ }^{1} \cdot$ Todd A. Schwartz ${ }^{3,4}$. Deborah K. Mayer ${ }^{1,4}$
}

Received: 4 January 2021 / Accepted: 8 March 2021 / Published online: 31 March 2021

(C) The Author(s), under exclusive licence to Springer-Verlag GmbH Germany, part of Springer Nature 2021

\begin{abstract}
Purpose The COVID-19 pandemic has exacerbated cancer treatment disparities, including accessibility to resources. We describe the process and outcomes of a new proactive, virtual nurse-led, resource center navigation model enhanced by using volunteer patient navigators. Using known patient risk factors, this model provides interventions to reduce barriers to care, with an emphasis on non-English-speaking populations.

Methods Patients were included if they (1) were in active cancer treatment and (2) had one or more known risk factors: distance from cancer hospital, needing complex care, 65 years or older, malignant hematological diagnosis, new treatment start, lives alone, non-English speaker, or a new hospital discharge. Nurse navigators triaged referrals to appropriate team members who identified and addressed barriers to care.

Results The program engaged with 586 adult cancer patients over 1459 encounters. The most common risk factors included distance (59.7\%), complex care (48.8\%), and new treatment start (43.5\%). The most common interventions were core education (69.4\%), emotional support (61.2\%), and education (35.7\%). Statistical differences were found between Spanish-speaking $(n=$ $118)$ and non-Spanish-speaking patients $(n=468)$. While Spanish-speaking patients had fewer risk factors (1.95 vs. $2.80, p \leq$ $.0001)$, they had nearly double the number of visits ( 4.27 vs. $2.04, p \leq .0001)$ and $69 \%$ more interventions ( 8.26 vs. $4.90, p \leq$ $.0001)$. Many patients (42.7\%) required follow-up visits.

Conclusion We successfully established a new navigation model for the resource center during the pandemic that identified and reduced barriers to care, particularly in the Spanish-speaking population.
\end{abstract}

Keywords Resource center $\cdot$ Volunteers $\cdot$ Risk factors $\cdot$ Patient navigation $\cdot$ Cancer $\cdot$ Spanish-speaking

\section{Introduction}

The COVID-19 pandemic is disproportionately affecting lowincome, minorities, and a broad range of other vulnerable

Sharon M. Bigelow

Sharon.bigelow@unchealth.unc.edu

1 Lineberger Comprehensive Cancer Center, North Carolina Cancer Hospital, Chapel Hill, NC, USA

2 University of North Carolina at Chapel Hill, Chapel Hill, NC, USA

3 Department of Biostatistics, Gillings School of Global Public Health, Chapel Hill, NC, USA

4 School of Nursing, University of North Carolina at Chapel Hill, Chapel Hill, NC, USA populations, including cancer patients [1]. Cancer patients are experiencing deferred treatments, delayed evaluation, interrupted ancillary services, and increases in barriers to care [1].

Navigation programs have been implemented to address inequitable access to cancer care [2,3]. The goals of most navigation programs include linking patients to primary care services, specialist care, community-based health, and social services, as well as identifying and addressing patient barriers to care. Patient navigation encompasses a wide range of community roles, including but not limited to nurses, social workers, and lay volunteers. Navigation programs are in an optimal position to address the pandemic's impact on cancer care $[4,5]$.

Prior to the pandemic, the North Carolina Cancer Hospital's (NCCH) Patient and Family Resource Center (PFRC) used a passive "retail" model of patient navigation, 
relying on help-seeking patients and families as well as referrals to connect patients with resources and interventions to reduce or eliminate their clinical and non-clinical barriers to care. To address the increased barriers to care during the pandemic, the PFRC adopted a novel navigation strategy, a proactive virtual resource center navigation program to focus on the needs of the state's most vulnerable cancer populations. With this new navigation model, patients were assessed to determine relevant risk factors for poorer outcomes to identify the most vulnerable cancer patients and provide targeted interventions to maximize clinical and non-clinical outcomes.

Our model, led by nurse navigators (NN) in the PFRC, is a patient-centric model comprising a multidisciplinary team of hospital staff (nurses, NNs, social workers, dietitians, and interpreters) and trained staff of volunteer patient navigators (VPN). With the addition of VPNs, the team's outreach capacity was able to expand by designating PFRC NNs as coordinators for volunteers to reach out to a larger cohort of eligible patients than would have been resourced by the existing retail model. In addition to their VPN training, VPNs had weekly meetings for the first 4 weeks of joining the program to ensure they were adequately onboarded and then monthly thereafter. The increase in volunteers additionally allowed for deepened relationship building and further patient-centered care to address disparities.

The purpose of this paper is to describe the implementation, associated interventions, and outcomes of the PFRC's proactive virtual resource center navigation model.

\section{Methods}

This report summarizes patient visits between 3/24/2020 and 10/23/2020 at NCCH, part of the Lineberger Comprehensive Cancer Center, an NCI-designated Comprehensive Cancer Center. The University of North Carolina at Chapel Hill Institutional Review Board and Office of Human Research Ethics provided exempt status (IRB\# 20-2432) for the collection and use of this de-identified patient data for this quality improvement project. An Excel database was used to capture referral information, patient demographics, risk characteristics, visit data, interventions, and outcomes. Patients were identified if they were in active treatment for a cancer diagnosis and concurrently had one or more of the outlined risk factors. Patients were not excluded from referral to this program based on gender, race, ethnic orientation, or sexual orientation.

\section{Risk factors for program eligibility}

Our first step was identifying patients with the highest need for navigation services. In 2019, the PFRC conducted over 100 interviews with cancer patients and their families to better understand their ongoing engagement with the PFRC as well as identify patient barriers to care. These interviews, supplemented with a literature review, informed our selection of risk factors and subsequent interventions. The following risk factors were identified and are described below: distance from cancer center, needing complex care, 65 years or older, malignant hematological diagnosis, new treatment start, lives alone, non-English speaker, and new hospital discharge.

Distance from cancer center: This was defined as a minimum of a 1-hour, one-way trip duration. Prior studies have indicated that a long distance from a respective healthcare facility has been associated with increased travel burden and worse progression-free survival and overall survival, especially for those with lower incomes or under/uninsured [6-8].

Complex care: Needing complex care was indicated by one or more of the following: combined modality treatment (surgery, radiation, chemotherapy, or other systemic treatments), metastatic disease, two or more comorbidities (e.g., diabetes, heart disease), stem cell transplant, CAR T-cell therapy, or diagnosed psychiatric disorder, which worsen health outcomes among cancer patients [9-11].

Sixty-five years or older: Older patients not only have an increased physiological burden from cancer but also frequently present with several comorbidities that have the potential to impact their ongoing cancer treatment negatively [12].

Malignant hematological diagnosis: This risk factor is based on evidence of poorer outcomes compared to solid tumor cancer patients [13]. A recent study provided evidence that patients with hematological malignancies and COVID-19 had a worse survival than both the general COVID-19 affected population and patients with hematological malignancies without COVID-19 [14].

New treatment start: New treatment, defined as beginning a new anticancer treatment within the past 30 days, is related to a lack of familiarity with a treatment plan, lack of knowledge about personal side-effect management, or when to call or come to the facility are common. Poorer initial outcomes may be experienced, including increased hospitalizations and emergency room use and reduced quality of life [15-17].

Lives alone: Individuals who live alone often have worse disease outcomes and reduced survival than those who do not live alone [18]. COVID-19 has exacerbated the physical isolation of living alone to include social isolation and make it more challenging to meet their basic physical, medical, and emotional needs [19].

Non-English speaker: Non-English-speaking cancer patients face difficulties communicating with their healthcare providers, are commonly being diagnosed at later stages, lack immediate access to translated cancer information, and cannot gain access to the care they need, often resulting in worse outcomes [20]. Non-English speakers were defined as individuals whose electronic medical record (EMR) was flagged as requiring translation services [21]. 
New hospital discharge: Patients who are newly discharged (within the previous 14 days of the support call) still require continued medical attention, care, and information. The transition to home or outpatient care may result in confusion and a lack of guidance for the patient leading to higher readmission risk. Of those discharged from the hospital and readmitted within 30 days, analyses identified $31 \%$ of readmissions to be potentially avoidable [22-24].

\section{Procedure}

Referrals for the program were generated in two ways. The first was a referral from the medical team or hospital staff who identified eligible patients. The second was driven by a PFRC nurse navigator $(\mathrm{NN})$, who reviewed those patients scheduled for hospital treatments each day to determine which patients met the program criteria. Once identified, an EMR in-basket email message was routed to an in-basket pool explicitly created for the navigation program.

The NN determined the complexity level based on the perceived needs and risk factors and then triaged to the appropriate team member (i.e., NN, VPN, or Spanish liaison (SL)). For example, transportation needs or food insecurity would be assigned to a VPN, while educating a patient on a disease or treatment would be assigned to a NN. The NN completed a referral form required for the team member to connect and intervene by a target call date (Electronic Supplementary Material). All communications sent and received were completed through secure channels, internal messaging, and documentation within the EMR to ensure HIPAA compliance.

The majority of visits were completed by phone or video, although limited staff at the PFRC were able to complete some in-person visits. Due to remote operations, the team required additional training to use online networking services. When conducting phone or video visits, an institution-approved medical platform was implemented which displayed the PFRC phone number. The navigator began the visit by verifying the patient's identity and then conducting a standardized barrier assessment using a script to ensure consistency among team members. The script addressed five key areas: financial, logistical, medical, emotional, and social barriers (Electronic Supplementary Material).

When an unmet need was identified, the VPN, NN, or SL addressed concerns with respective interventions including, but not limited to, food pantry resources, financial relief resources, patient portal technical assistance, transportation resources, social worker requests, external referrals, caregiver support, advanced directives education and preparation, and emotional support. Core education regarding COVID-19, the PFRC, and after hours were included in all of the interventions. The interventions were either educational or referrals for an internal resource or a vetted external resource. External resources have been prone to closure during the pandemic, so it was essential that an updated resource list was maintained, quality-checked, and easily accessed by team members through a shared portal to ensure that the patients were given the most up-to-date, comprehensive, and accurate referrals. Ultimately, if unaddressed medical concerns remained, the $\mathrm{NN}$ referred the patient back to their clinical team.

Once the visit was completed, the navigators securely submitted a report form detailing the visit, barriers addressed, relevant interventions, and if further action was required. The report was then filed at the PFRC, and a summary was uploaded into the patient's EMR for documentation. If the need was medically based, such as unresolved symptoms or side effects, the issue was immediately routed to the $\mathrm{NN}$, who alerted the medical team to any urgent or unresolved needs. The role of the NN was to ensure that all barriers were addressed and resolved promptly to the extent achievable. Frequently, follow-up visits were necessary to complete all of the interventions required to overcome the barriers to care.

The visit outcomes were described using the following analysis. Descriptive statistics were computed as frequencies and percentages for the risk factors at the patient level and the interventions at the visit level. Patients were additionally classified by Spanish-speaking status; since the size of the population that did not speak English or Spanish was small $(n=8)$, the analysis of non-English-speaking patients focused solely on Spanish-speaking patients.

Fisher's test was used to compare these percentages for Spanish-speaking patients versus non-Spanish-speaking patients. Means and standard deviations were computed to summarize the number of risk factors, the number of visits at the patient level, and the number of interventions at the visit level. The groups were compared for these variables using a Wilcoxon rank-sum test.

\section{Results}

There were 657 patients referred to the program and 586 patients engaged in 1459 completed visits (Table 1). The remaining $10.9 \%(n=71)$ of patients never answered the phone, returned messages, nor connected with the staff. Of the 1459 visits, 14 VPNs completed 44.5\% ( $n=650), 3$ SLs completed $33.7 \%(n=491)$, and 3 NNs completed $21.8 \%(n=318)$ of the visits. The racial demographics of the 586 patients are described in Table 1 with $45.9 \%(n=269)$ White, $24.9 \%(n$ $=146)$ Black, $1.2 \%(n=7)$ Asian, $0.2 \%(n=1)$ Native American, and 26.4\% $(n=155)$ Other. Many who selected "Other" were presumed to be Latinx as they were identified as Spanish-speaking $(n=107)$ in the EMR. English-speaking patients were 78.5\% $(n=460)$, while non-English-speaking patients were $21.5 \%(n=126)$. There were more females $(60.1 \%, n=352)$ than males $(39.6 \%, n=232)$, and $41.1 \%$ $(n=241)$ of patients were 65 years or older. Regarding health 
Table 1 Patient demographics $(N=586)$

\begin{tabular}{|c|c|c|}
\hline Characteristics & $N$ & $\%$ \\
\hline \multicolumn{3}{|l|}{ Age } \\
\hline $18-39$ & 71 & $12.1 \%$ \\
\hline $40-64$ & 274 & $46.8 \%$ \\
\hline $65+$ & 241 & $41.1 \%$ \\
\hline \multicolumn{3}{|l|}{ Gender } \\
\hline M & 233 & $39.8 \%$ \\
\hline $\mathrm{F}$ & 353 & $60.2 \%$ \\
\hline \multicolumn{3}{|l|}{ Race } \\
\hline White & 270 & $46.1 \%$ \\
\hline Black & 147 & $25.1 \%$ \\
\hline Asian & 7 & $1.2 \%$ \\
\hline Native & 1 & $0.2 \%$ \\
\hline Latinx & 6 & $1.0 \%$ \\
\hline Other & 155 & $26.4 \%$ \\
\hline \multicolumn{3}{|l|}{ Language } \\
\hline English & 460 & $78.5 \%$ \\
\hline Non-English & 126 & $21.5 \%$ \\
\hline \multicolumn{3}{|l|}{ Insurance } \\
\hline Employer/private & 167 & $28.5 \%$ \\
\hline Medicare only & 21 & $3.6 \%$ \\
\hline Medicare + supplement & 187 & $31.9 \%$ \\
\hline Medicaid only & 64 & $10.9 \%$ \\
\hline Medicare + Medicaid & 36 & $6.2 \%$ \\
\hline No insurance & 111 & $18.9 \%$ \\
\hline \multicolumn{3}{|l|}{ Diagnosis } \\
\hline Breast & 88 & $15.0 \%$ \\
\hline Central nervous system & 7 & $1.2 \%$ \\
\hline Gastrointestinal & 69 & $11.8 \%$ \\
\hline Genitourinary & 30 & $5.1 \%$ \\
\hline Gynecological & 59 & $10.1 \%$ \\
\hline Hematological & 190 & $32.4 \%$ \\
\hline Head and neck & 50 & $8.5 \%$ \\
\hline Lung & 62 & $10.6 \%$ \\
\hline Melanoma & 6 & $1.0 \%$ \\
\hline Others & 25 & $4.3 \%$ \\
\hline
\end{tabular}

insurance, $28.4 \%$ of patients were covered by employer/ private insurance; $52.2 \%$ had Medicaid + Medicare, Medicare only, and/or supplemental insurance; and 18.6\% did not have insurance. The most common cancer diagnoses were hematological $(32.4 \%, n=190)$, breast $(15.0 \%, n=88)$, and gastrointestinal $(11.8 \%, n=69)$ malignancies.

Based on our barrier assessment, the risk factors most commonly identified included those living at a distance (59.7\%), needing complex care $(48.8 \%)$, or having a new treatment start (43.5\%) (Fig. 1).

Interventions were categorized by type in our analysis: advanced directives (education and preparation); MyChart (patient portal education and signup); financial (lodging, pharmacy financial, general monies, gas card program, COVID relief monies, and financial navigation); education (food insecurity, pharmacy medications, nutrition information, diagnosis, and PFRC miscellaneous); emotional support (emotional support and caregiver support); and core education (PFRC, COVID-19, and after hours). All interventions not categorized into these groups (adolescent young adult program referral, psycho-oncology referral, transportation information, medical care team referral, SW referral, oncology dietitian referral, external referral, get real \& heel or yoga program, health coaching, palliative care referral) were treated as individual interventions and are illustrated in Fig. 2.

The five most common interventions categories included core education (69.4\%), emotional support (61.2\%), education $(35.7 \%)$, financial $(26.4 \%)$, and MyChart (17.3\%) (Fig. 2).

We noted important differences between Spanish-speaking $(n=118)$ and non-Spanish-speaking patients $(n=468)$. Spanish-speaking patients were less likely to be receiving complex care $(p \leq .0001), 65$ years or older $(p \leq .0001)$, or lives alone $(p=.0053)$ (Fig. 3). Non-Spanish-speaking patients received oncology dietitian referrals $(p \leq .0001)$, external referrals $(p \leq .0001)$, emotional support $(p \leq .0001)$, and education $(p=.0003)$ at a higher frequency than Spanishspeaking patients. Spanish-speaking patients received MyChart interventions $(p \leq .0001)$ and core education $(p \leq$ $.0001)$ at a higher frequency than non-Spanish-speaking patients (Fig. 4). Spanish-speaking patients had fewer risk factors (1.95 vs. $2.80, p \leq .0001)$ but nearly double the number of visits $(4.27$ vs. $2.04, p \leq .0001)$ and $69 \%$ more interventions per patient (8.26 vs. $4.90, p \leq .0001)$ compared to nonSpanish-speaking patients (Fig. 5).

Although the assessment was intended as a one-time visit, many patients required a follow-up visit $(42.7 \%, n=250)$ to complete addressing their needs; $9.4 \%(n=55)$ of patients required five or more visits, with the majority of these patients being Spanish-speaking.

\section{Discussion}

As our PFRC partially closed in March 2020 during the beginning of the COVID-19 pandemic, the previous "retail" navigation model was unable to meet the needs of cancer patients and families. The majority of the PFRC staff shifted to remote work, and volunteers were sidelined from their usual clinic navigation tasks causing many patients to be deferred from onsite assessment and intervention. Therefore, the decision to restructure the VPN role for this virtual model was efficient and effective. Because VPNs were unpaid, no additional costs were incurred. This updated proactive virtual resource center 
Fig. 1 Percentage of patients $(n=$ 586 ) with risk factors

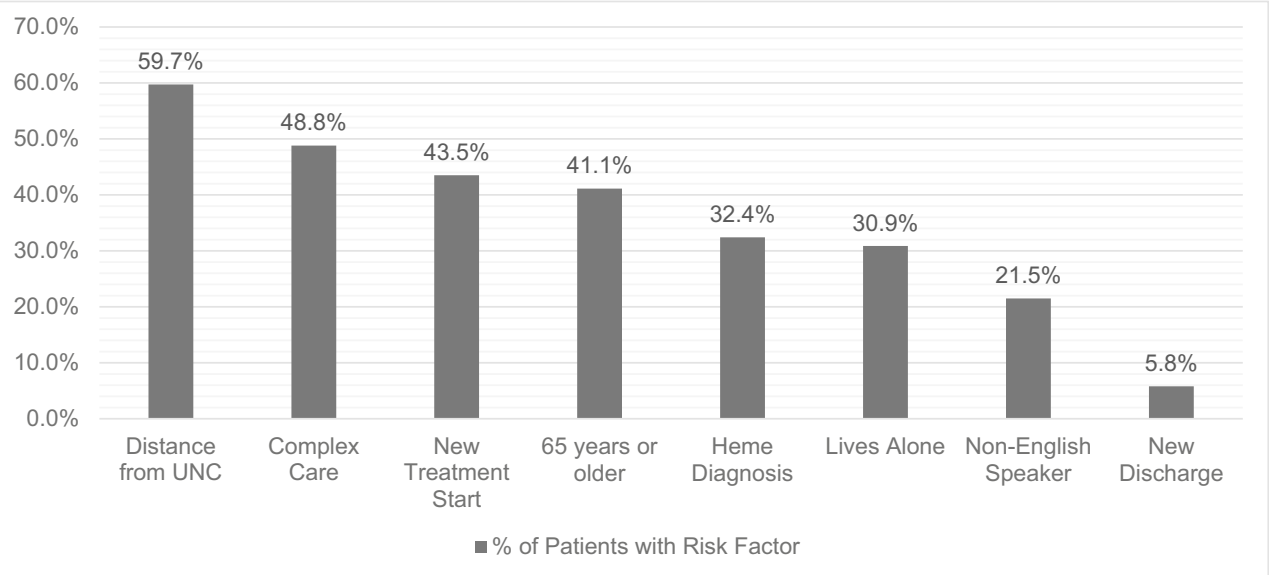

navigation model provided support and improved the care and safety of 586 patients and families over 1459 visits who may not have otherwise received these services during the pandemic. Based on the data collected per visit on the number of risk factors and interventions, the results demonstrated that the PFRC successfully addressed barriers to care.

The most frequent risk factors identified were distance from cancer center, needing complex care, and new treatment start (Fig. 1). This program provided an opportunity to substantiate further the need to address these barriers to care by extending our reach to a more distant and complex patient audience.

Barrier assessments indicated that many patients were unaware of their needs, and previous PFRC engagement interviews demonstrated that even those who were aware of their needs were unaware of the resources available to them. In addition, the navigators observed that not all of the unmet needs were due to the pandemic. Some faced pre-existing barriers (e.g., mounting bills due to lack of insurance), while others experienced barriers exacerbated by the pandemic (e.g., financial hardship from job loss or increased need for emotional support and education). As a result, the frequency of interventions such as emotional support, financial, and education was increased to meet these patient needs (Fig. 2). In addition, the pandemic uniquely impacted cancer patients and families, requiring additional education beyond what the general public receives (e.g., heightened safety measures due to increased risk to COVID-19 morbidity and mortality in immunocompromised individuals) [25]. As a result, a new COVID-19 education intervention was developed and delivered to patients as part of the core education.

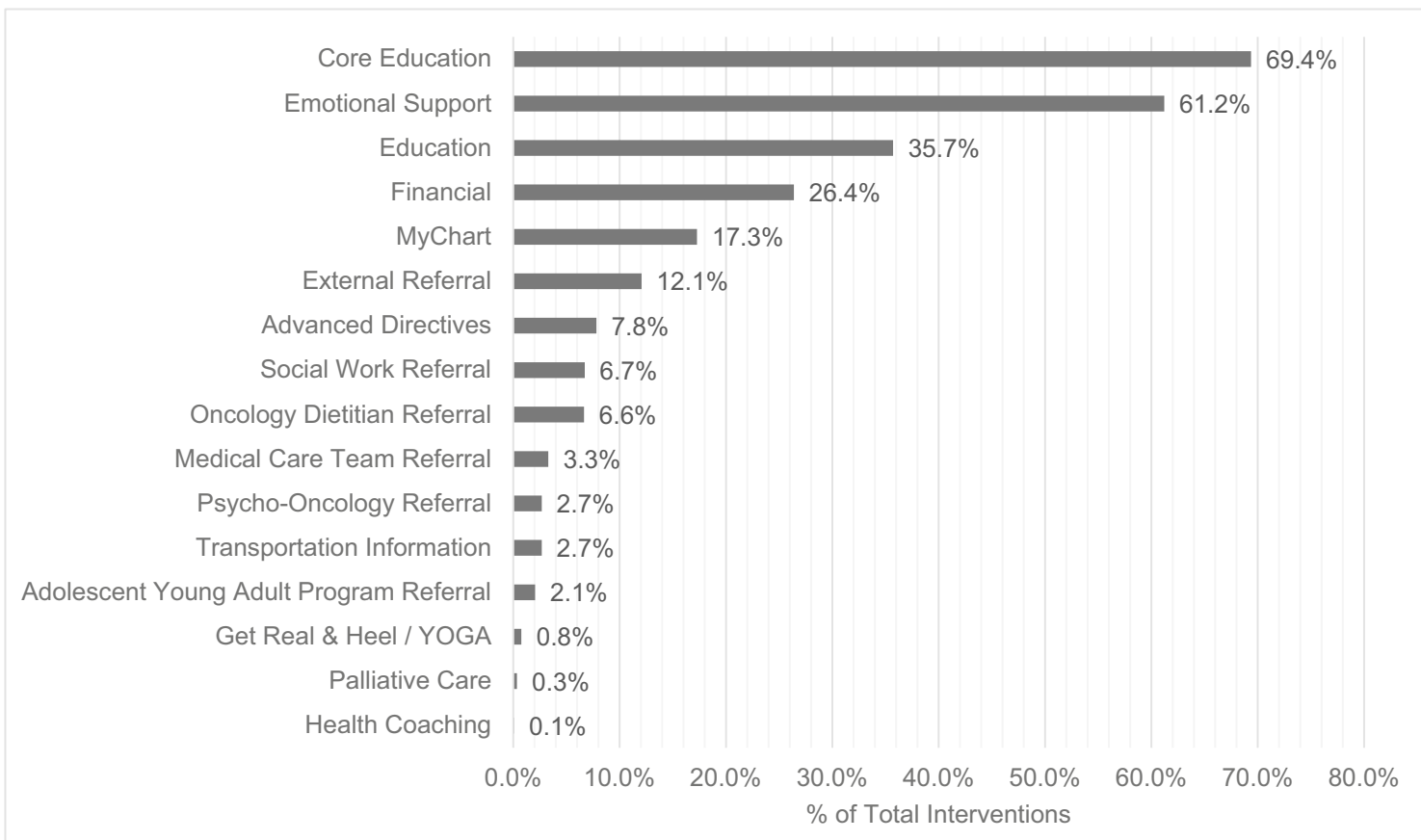

Fig. 2 Total intervention frequency (grouped) across all visits ( $n=1459$ completed visits) 
In stratifying the patient population by risk factors, comparing Spanish-speaking patients vs. non-Spanish-speaking patients produced findings of interest to the PFRC. First, Spanish-speaking patients were more likely to live with family, less likely to be 65 years or older, and less likely to have complex care needs than non-Spanish-speaking patients (Fig. 3). Second, to reduce the knowledge gap between Spanishspeaking and non-Spanish-speaking patients, Spanishspeaking patients required more frequent and repeated instruction on navigating the fundamentals (core education and MyChart) of their visit at significantly higher frequencies than non-Spanish-speaking patients. In contrast, non-Spanishspeaking patients received emotional support, external referral, oncology dietitian referral, and education at significantly higher frequencies than Spanish-speaking patients, indicating that they exhibited a strong understanding of the fundamentals of their visit and managing their disease during the pandemic (Fig. 4).

Additionally, Spanish-speaking patients had significantly fewer risk factors but required almost twice the number of visits and about two-thirds more of the interventions than nonSpanish-speaking patients, all of which were statistically and clinically significant findings (Fig. 5). Three potential reasons may provide an explanation. First, that speaking Spanish is in and of itself a sufficient risk factor and driver of barriers and interventions even in the absence of other risk factors. Second, the selected risk factors used in our model are insufficient to describe the full lived experience and barriers that Spanishspeaking cancer patients face. Third, translation between English and Spanish could have been a barrier to identifying all unmet needs. Creating a more culturally diverse volunteer cohort and workforce could provide improved insight into this population. Furthermore, the program must continue to research what the primary drivers of the disparities in this population are.

We shared our experiences with other UNC affiliated community cancer sites. As a result, two sites (McCreary Cancer Center of Caldwell UNC Health Care and UNC Lenoir Health Care Cancer Center) established similar programs based on this model. These sites utilized four volunteers to make 223 calls (186 and 37 calls at McCreary and Lenoir, respectively) to 124 patients (102 and 22 patients at McCreary and Lenoir, respectively) over 4 months. These sites reported satisfaction with the program's ability to address patient barriers during the implementation of new pandemic guidelines while maintaining their volunteers in a meaningful way. Other network sites are also considering adoption of this virtual program.

\section{Limitations}

This program did not engage with the entire $\mathrm{NCCH}$ population due to our referral and selection process. In addition, the navigation program was unable to connect with $10.8 \%$ of the referred patients, defined as not answering after three attempted calls on two separate days. The inability to connect was likely due to patients' unfamiliarity with our virtual navigation program and our methods for contacting them.

This program was not culturally competent enough to meet the needs of all non-English speakers, given that most navigators needed to rely on interpreters for communication with this population. Outsourced or staff interpreters were used for six languages at $\mathrm{NCCH}$ : Korean, Nepali, Romanian, Ukrainian, Vietnamese, and Spanish, with Spanish-speaking
Fig. 3 Percentage of patients with risk factors: Spanish-speaking ( $n$ $=118$ ) and non-Spanish-speaking $(n=468)(* * * * p$ value $<0.0001$, $* * * p$ value $<.001)$

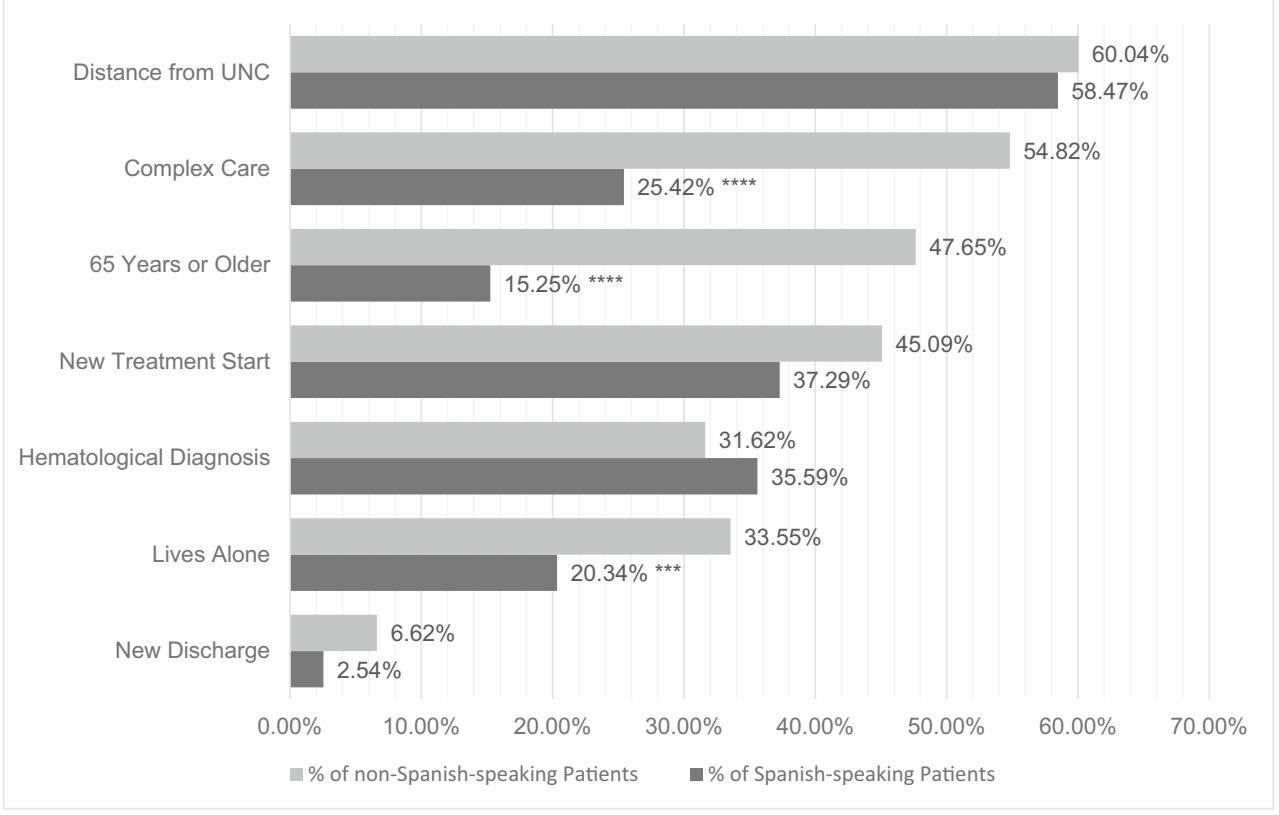




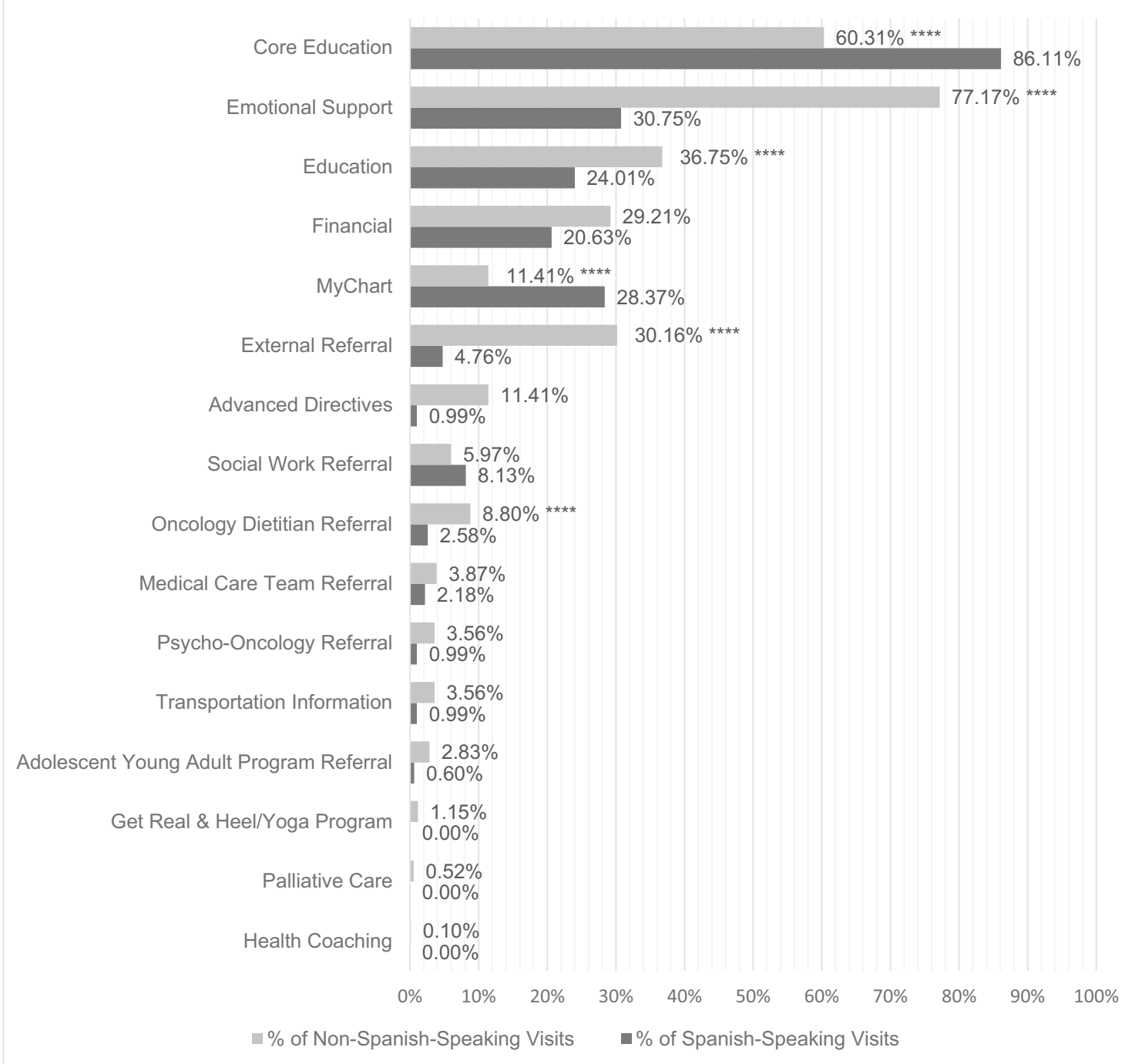

Fig. 4 Interventions provided during Spanish-speaking visits $(n=504)$ and non-Spanish-speaking visits $(n=955)(* * * * p$ value $<0.0001)$

being the most utilized. While necessary, the use of interpretation services added an extra layer of communication, which

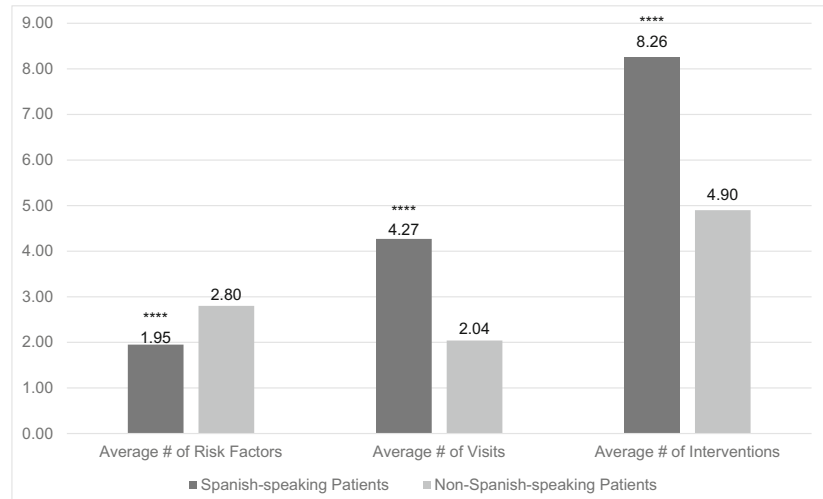

Fig. 5 Risk factors, visits, and interventions per patient for Spanishspeaking $(n=118)$ and non-Spanish-speaking $(n=468)$ patients $(* * * * p$ value $<0.0001)$ may have reduced the identification and provision of interventions such as emotional support.

While the model initially included distress as a risk factor for high utilization of resources, the authors acknowledged the difficulty of accurately assessing distress during multiple virtual patient interactions and removed distress as a risk factor. However, distress continued to be addressed within the standard scripting, and appropriate referrals were made as needed.

The program was initially delivered only to one hospital, which had resources such as a robust student and community volunteer program that may not be available at community hospitals. Having two network sites implement programs based on this model adds some reproducibility; however, the bandwidth and sustainability of this volunteer force have yet to be fully determined.

The VPN position has limitations in the scope of practice, such as the inability to access and use the EMR, which restricts the level of understanding of the VPN regarding patient clinical and socioeconomic barriers. 


\section{Future research}

Further exploration of risk factors as a driver of interventions, stratification of patients by risk factors, and earlier identification of patients along the continuum of care is necessary for the continued reduction of barriers to cancer care in our navigation model. Immediate additions to the model would include distress screenings in an outpatient navigation program and mapping patient residency with population health outcomes of counties with long-standing poverty, which is associated with increased cancer morbidity and mortality [26].

Given that this paper illuminated significant differences between Spanish-speaking patients and non-Spanishspeaking patients, it is imperative that future research critically analyze these differences to improve outcomes in the Spanishspeaking population $[27,28]$.

It will be essential to investigate further the reliability of risk factors in determining interventions, program's cost-effectiveness, staff cultural competency, and whether the employment status of current VPNs will produce the most significant patient gains. Future research is necessary to evaluate this and other navigation models' effectiveness on patient outcomes, such as quality of life and acute care utilization, metrics that were not incorporated in this initial model.

\section{Conclusion}

We successfully transitioned to a new proactive, virtual outreach program to educate, advocate for, resource, and support our cancer patients during the COVID-19 pandemic. The hybrid program accommodated both remote and onsite visits for patients and staff, making it resilient against disruption and increasing engagement in higher-risk patients compared to our previous retail model. Utilizing VPNs was critical in reducing barriers to cancer care delivery, particularly in our Spanishspeaking population. While our findings are specific to the NCCH PFRC navigation model, these findings are being used to inform the development of a system-wide comprehensive navigation model. Additional investigation is necessary to understand the sustainability and practicality of future models, the longevity of the volunteer workforce, and the program's impact on patient outcomes after the pandemic. With increasingly limited resources, a high-value, comprehensive, patientcentric navigation model is essential to promote optimal cancer patient care outcomes.

Supplementary Information The online version contains supplementary material available at https://doi.org/10.1007/s00520-021-06147-3.

Acknowledgements We would like to acknowledge Matthew Washko, University of North Carolina, Crystal Dula, RN, McCreary Cancer Center, and Megan Evans, OSW, UNC Lenoir, for their contributions to this manuscript from their respective programs.
Availability of data and materials Original data is available upon request.

Code availability Original SAS® code is available upon request.

Author contribution All authors contributed to the planning and execution of this program as well as the writing, reading, and approval of the final manuscript.

Funding Deborah K. Mayer, Ph.D., RN, AOCN®, FAAN receives partial support from the Duke Endowment Fund Grant 6650-SP.

\section{Declarations}

Ethics approval This is a quality improvement study. The Office of Human Research Ethics at the University of North Carolina has confirmed that the Institutional Review Board approval is not required (IRB 20-2432).

Consent to participate Not applicable

Consent for publication Not applicable

Conflicts of interest The authors declare no competing interests.

\section{References}

1. Hanna TP, King WD, Thibodeau S, Jalink M, Paulin GA, HarveyJones E, O'Sullivan DE, Booth CM, Sullivan R, Aggarwal A (2020) Mortality due to cancer treatment delay: systematic review and meta-analysis. BMJ 371:m4087. https://doi.org/10.1136/bmj. $\mathrm{m} 4087$

2. Freeman HP, Rodriguez RL (2011) History and principles of patient navigation. Cancer 117(15 Suppl):3539-3542. https://doi.org/ 10.1002/cncr.26262

3. Lopez D, Pratt-Chapman ML, Rohan EA, Sheldon LK, BasenEngquist K, Kline R, Shulman LN, Flores EJ (2019) Establishing effective patient navigation programs in oncology. Support Care Cancer 27(6):1985-1996. https://doi.org/10.1007/s00520-01904739-8

4. Tho PC, Ang E (2016) The effectiveness of patient navigation programs for adult cancer patients undergoing treatment: a systematic review. JBI Database System Rev Implement Rep 14(2):295321. https://doi.org/10.11124/jbisrir-2016-2324

5. Wells KJ, Campbell K, Kumar A, Clark T, Jean-Pierre P (2018) Effects of patient navigation on satisfaction with cancer care: a systematic review and meta-analysis. Support Care Cancer 26(5): 1369-1382. https://doi.org/10.1007/s00520-018-4108-2

6. Barrington DA, Dilley SE, Landers EE, Thomas ED, Boone JD, Straughn JM Jr, McGwin G Jr, Leath CA 3rd (2016) Distance from a comprehensive cancer center: a proxy for poor cervical cancer outcomes? Gynecol Oncol 143(3):617-621. https://doi.org/10. 1016/j.ygyno.2016.10.004

7. National Academies of Sciences E, Medicine (2018) Health-care utilization as a proxy in disability determination. The National Academies Press, Washington, DC. https://doi.org/10.17226/ 24969

8. Syed ST, Gerber BS, Sharp LK (2013) Traveling towards disease: transportation barriers to health care access. J Community Health 38(5):976-993. https://doi.org/10.1007/s10900-013-9681-1 
9. Aquina CT, Mohile SG, Tejani MA, Becerra AZ, Xu Z, Hensley BJ, Arsalani-Zadeh R, Boscoe FP, Schymura MJ, Noyes K, Monson JR, Fleming FJ (2017) The impact of age on complications, survival, and cause of death following colon cancer surgery. Br J Cancer 116(3):389-397. https://doi.org/10.1038/bjc.2016.421

10. Smith AW, Reeve BB, Bellizzi KM, Harlan LC, Klabunde CN, Amsellem M, Bierman AS, Hays RD (2008) Cancer, comorbidities, and health-related quality of life of older adults. Health Care Financ Rev 29(4):41-56

11. Sogaard M, Thomsen RW, Bossen KS, Sorensen HT, Norgaard M (2013) The impact of comorbidity on cancer survival: a review. Clin Epidemiol 5(Suppl 1):3-29. https://doi.org/10.2147/CLEP. S47150

12. Sarfati D, Koczwara B, Jackson C (2016) The impact of comorbidity on cancer and its treatment. CA Cancer J Clin 66(4):337-350. https://doi.org/10.3322/caac.21342

13. Vijenthira A, Gong IY, Fox TA, Booth S, Cook G, Fattizzo B, Martin Moro F, Razanamahery J, Riches JC, Zwicker JI, Patell R, Vekemans M-CM, Scarfo L, Chatzikonstantinou T, Yildiz H, Lattenist R, Mantzaris I, Wood WA, Hicks LK (2020) Outcomes of patients with hematologic malignancies and COVID-19: A systematic review and meta-analysis of 3377 patients. Blood. 136: 2881-2892. https://doi.org/10.1182/blood.2020008824

14. Passamonti F, Cattaneo C, Arcaini L, Bruna R, Cavo M, Merli F, Angelucci E, Krampera M, Cairoli R, Della Porta MG, Fracchiolla N, Ladetto M, Gambacorti Passerini C, Salvini M, Marchetti M, Lemoli R, Molteni A, Busca A, Cuneo A, Romano A, Giuliani N, Galimberti S, Corso A, Morotti A, Falini B, Billio A, Gherlinzoni F, Visani G, Tisi MC, Tafuri A, Tosi P, Lanza F, Massaia M, Turrini M, Ferrara F, Gurrieri C, Vallisa D, Martelli M, Derenzini E, Guarini A, Conconi A, Cuccaro A, Cudillo L, Russo D, Ciambelli F, Scattolin AM, Luppi M, Selleri C, Ortu la Barbera E, Ferrandina C, di Renzo N, Olivieri A, Bocchia M, Gentile M, Marchesi F, Musto P, Federici AB, Candoni A, Venditti A, Fava C, Pinto A, Galieni P, Rigacci L, Armiento D, Pane F, Oberti M, Zappasodi P, Visco C, Franchi M, Grossi PA, Bertù L, Corrao G, Pagano L, Corradini P, ITA-HEMA-COV Investigators (2020) Clinical characteristics and risk factors associated with COVID-19 severity in patients with haematological malignancies in Italy: a retrospective, multicentre, cohort study. Lancet Haematol 7(10):e737-e745. https://doi.org/10.1016/S2352-3026(20)30251-9

15. Xiao H, Quan H, Pan S, Y in B, Luo W, Tang M, Ouyang Y, Tang W (2018) Incidence, causes and risk factors for 30-day readmission after radical gastrectomy for gastric cancer: a retrospective study of 2,023 patients. Sci Rep 8(1):10582. https://doi.org/10.1038/ s41598-018-28850-8

16. Rudd RE (2019) Health literacy considerations for a new cancer prevention initiative. Gerontologist 59(Suppl 1):S7-S16. https:// doi.org/10.1093/geront/gnz032

17. Halbach SM, Enders A, Kowalski C, Pfortner TK, Pfaff H, Wesselmann S, Ernstmann N (2016) Health literacy and fear of cancer progression in elderly women newly diagnosed with breast cancer-a longitudinal analysis. Patient Educ Couns 99(5):855-862. https://doi.org/10.1016/j.pec.2015.12.012

18. Cavalli-Bjorkman N, Qvortrup SS, Pfeiffer P, Wentzel-Larsen T, Glimelius B, Sorbye H (2012) Lower treatment intensity and poorer survival in metastatic colorectal cancer patients who live alone. Br J Cancer 107(1):189-194. https://doi.org/10.1038/bjc.2012.186

19. Hwang TJ, Rabheru K, Peisah C, Reichman W, Ikeda M (2020) Loneliness and social isolation during the COVID-19 pandemic. Int Psychogeriatr 32(10):1217-1220. https://doi.org/10.1017/ S1041610220000988

20. Tan L, Gallego G, Nguyen TTC, Bokey L, Reath J (2018) Perceptions of shared care among survivors of colorectal cancer from non-English-speaking and English-speaking backgrounds: a qualitative study. BMC Fam Pract 19(1):134. https://doi.org/10. 1186/s12875-018-0822-6

21. Qureshi MM, Romesser PB, Jalisi S, Zaner KS, Cooley TP, Grillone G, Kachnic LA, Truong MT (2014) The influence of limited English proficiency on outcome in patients treated with radiotherapy for head and neck cancer. Patient Educ Couns 97(2):276282. https://doi.org/10.1016/j.pec.2014.07.031

22. Ray EM, Hinton SP, Reeder-Hayes KE (2019) Risk factors for healthcare utilization in patients with newly diagnosed advanced lung cancer. J Clin Oncol 37(27 suppl):111. https://doi.org/10. 1200/JCO.2019.37.27_suppl.111

23. Donze J, Aujesky D, Williams D, Schnipper JL (2013) Potentially avoidable 30-day hospital readmissions in medical patients: derivation and validation of a prediction model. JAMA Intern Med 173(8):632-638. https://doi.org/10.1001/jamainternmed.2013. 3023

24. Donze JD, Lipsitz S, Schnipper JL (2017) Risk factors and patterns of potentially avoidable readmission in patients with cancer. $\mathrm{J}$ Oncol Pract 13(1):e68-e76. https://doi.org/10.1200/JOP.2016. 011445

25. Konstantinos T, Gavriatopoulou M, Schizas D et al (2020) Oncology during the COVID-19 pandemic: challenges, dilemmas and the psychosocial impact on cancer patients (review). Oncol Lett 20:441-447. https://doi.org/10.3892/ol.2020.11599

26. Jayasekera J, Onukwugha E, Cadham C, Harrington D, Tom S, Pradel F, Naslund M (2019) An ecological approach to monitor geographic disparities in cancer outcomes. PLoS One 14(6): e0218712. https://doi.org/10.1371/journal.pone.0218712

27. Karliner LS, Kim SE, Meltzer DO, Auerbach AD (2010) Influence of language barriers on outcomes of hospital care for general medicine inpatients. J Hosp Med 5(5):276-282. https://doi.org/10.1002/ jhm. 658

28. Al Shamsi H, Almutairi AG, Al Mashrafi S, Al Kalbani T (2020) Implications of language barriers for healthcare: a systematic review. Oman Med J 35(2):e122. https://doi.org/10.5001/omj.2020. 40

Publisher's note Springer Nature remains neutral with regard to jurisdictional claims in published maps and institutional affiliations. 